\title{
The importance of particular coronary collaterals' pathway in acute coronary syndrome
}

\author{
Wesam A. Alhejily \\ Department of Medicine, King Abdullaziz University, Jeddah, Saudi Arabia
}

\section{Case report}

A 66-year-old woman presented with rapidly progressive chest pain. Her medical history was positive for a long-standing diabetes mellitus type 2 , hypertension, and dyslipidemia. Her initial workup showed diffuse

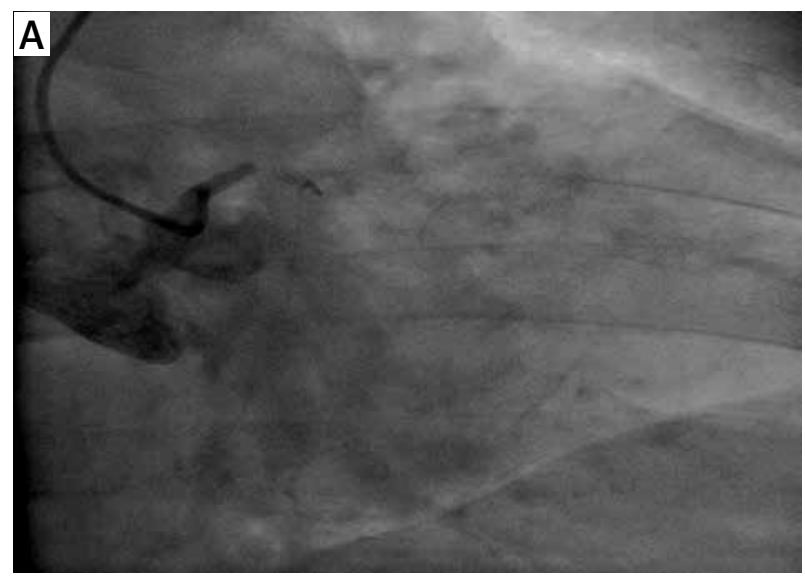

\section{c}

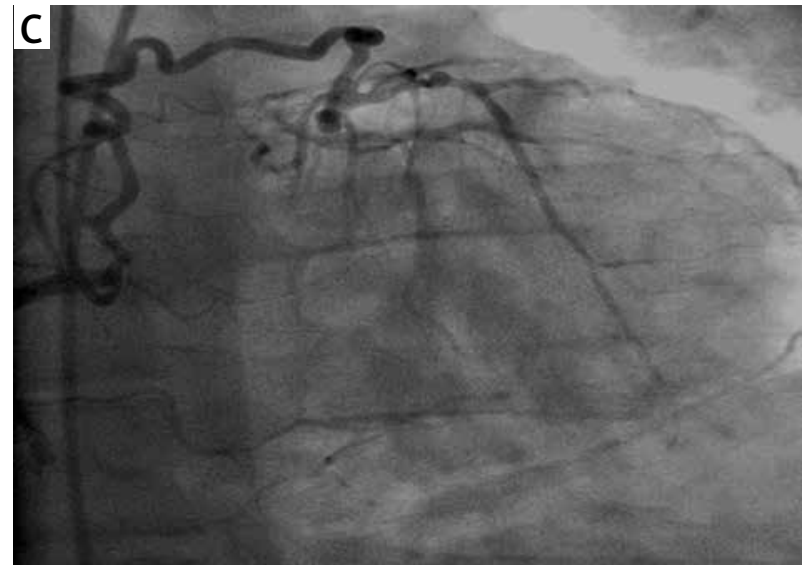

ST-segment depressions on a 12-lead ECG, and positive biomarkers including troponin I (Tn-I) $4.2 \mathrm{ng} / \mathrm{ml}$ (upper normal limit (UNL) $0-0.15 \mathrm{ng} / \mathrm{ml}$ ), creatine kinase (CK) 254 U/I (UNL 176) and creatine kinase MB (CKMB) 14 U/I (UNL 4 U/l). She underwent an urgent coronary angiogram.
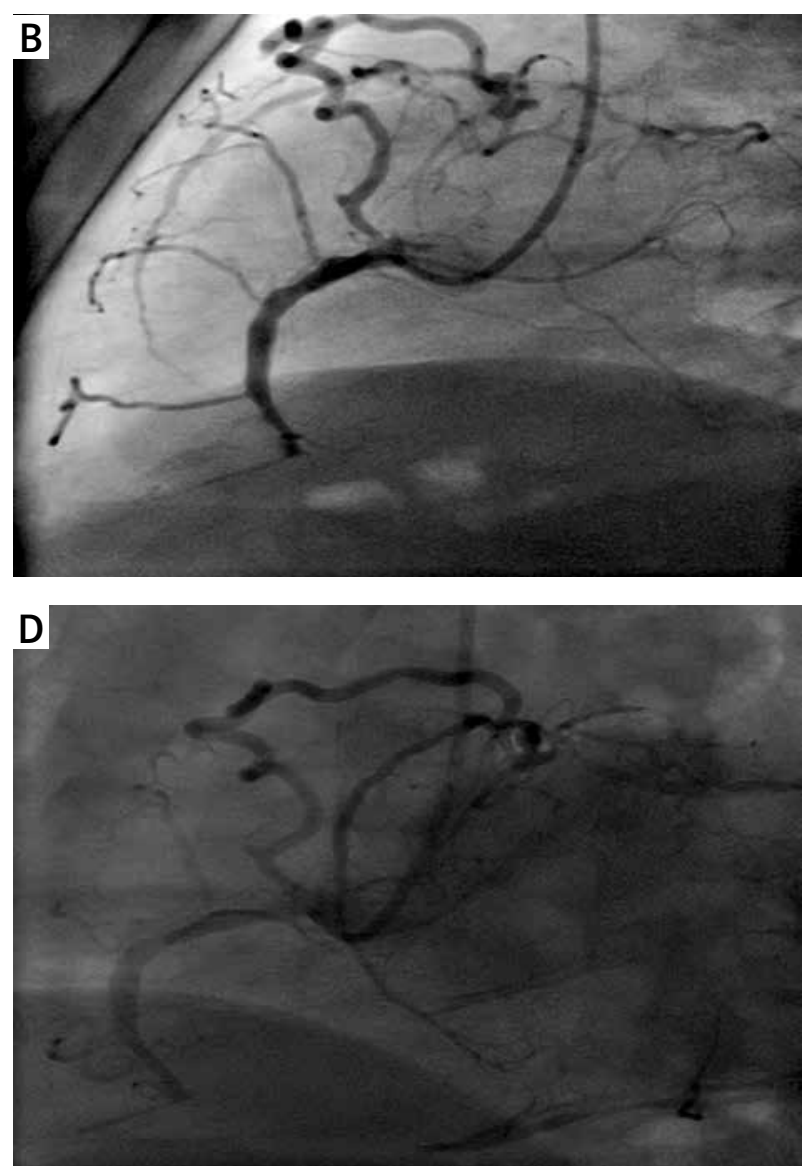

Figure 1. Panel A demonstrates an AP caudal view with flush occlusion of the distal left main; the LAD and LCX arteries are occluded, but LCX has a short segment of its proximal bed that is the partially recanalized (culprit) vessel. Panel $\mathbf{B}$ outlines the RCA and Vieussen's ring and its pathway supplying the entire left system. Panels $\mathbf{C}$ and $\mathbf{D}$ show distal collaterals from LAD and LCX to the distal RCA bed

\section{Corresponding author:}

Wesam A. Alhejily MD, FRCPC, FACP, FACC, FSCAl, Department of Medicine, King Abdullaziz University, PO Box 80215, Jeddah 21589,

Saudi Arabia, phone: +96 6546033322, e-mail: dr.wesam.h@gmail.com

Received: 20.12.2014, accepted: 22.02.2015. 
Four projections are shown in Figure 1 . In the antero-posterior caudal view (panel A), the left main artery is patent and well visualized but the left anterior descending (LAD) is not visible due to ostial occlusion. The culprit vessel was thought to be the left circumflex (LCX) artery; it is faintly seen as a partially recanalized short segment at its proximal bed. In the lateral projection (panel B) the right coronary artery (RCA) is seen at the left hand side and it is occluded at the mid segment; furthermore there is a conus branch off the RCA that has a long curvilinear course of an incomplete ring; it spans around the right ventricular outflow tract and the aorto-pulmonary trunk; and provides essential collaterals to the LAD and the LCX arteries. This finding is consistent with "Vieussen's ring" [1]. Panel C and D shows right and left anterior oblique projections of the collateral pathways to the $L A D$ and the $L C x$ arteries; there are also distal collaterals from the LAD and LCX to the RCA bed. The patient underwent successful coronary artery bypass surgery to the LAD utilizing the left internal mammary artery, and to the LCX and RCA arteries with saphenous vein grafting.

Vieussen's ring is an arterial collateral pathway between the right coronary artery conus branch and the left system. It is of paramount importance to adequately visualize the conus branch when injecting the right coronary artery to be able to see the entire course of the collateral and its pathway. In this particular case, it plays a major role, as it is the main blood supply to the entire left system and it made the decision of revascularization with bypass surgery feasible due to adequate targets seen only after injecting the right system; despite the well-established description of this particular type of collateral in the literature, we could not identify a single case report of such a finding in acute coronary syndrome patients. It can be detected incidentally in an otherwise normal coronary angiogram and usually it has no clinical significance. However, there have been reported cases of ruptured aneurysm of the ring leading to cardiac tamponade [2]. The aneurysm can be an incidental finding of a mass in a routine chest X-ray or a computed tomography scan $[3,4]$.

\section{Conflict of interest}

The author declares no conflict of interest.

\section{References}

1. O'Leary EL, Garza L, Williams M, et al. Vieussen's ring. Circulation 1998; 98: 487-8.

2. Owen AR, Moten SC, Molan MP. Rupture of an aneurysm of Vieussens' arterial ring presenting as acute cardiac tamponade. Clin Radiol 2009; 64: 1129-31.

3. Chan CY, Lee PW, Lam YY, et al. Images in cardiovascular medicine. Detection of large aneurysm of Vieussens' arterial ring after abnormal shadow on chest radiography. Circulation 2009; 120: e134-6.

4. Gupta V, Palacios IF, Aggarwal K. Anurysm of the Vieussens' arterial ring. J Invasive Cardiol 2007; 19: 282-3. 